\title{
Nitric Oxide: From Good to Bad
}

\author{
Paul M. Vanhoutte, MD, PhD
}

This essay summarizes a lecture presented on October 19th, 2017, during the 58th Annual Meeting of the Japanese College of Angiology in Nagoya, Japan. The lecture summarizes several instances where the absence of relaxations of isolated blood vessels in response to endothelium-dependent vasodilator agonists, which cause activation of endothelial nitric oxide synthase (eNOS) and consequent production of endothelium-derived nitric oxide (NO) and stimulation of soluble guanylyl cyclase (sGC) in underlying vascular smooth muscle, or hypoxia are curtailed or reversed to endothelium-dependent contractions. Chosen examples include selective dysfunction of eNOS activation in regenerated endothelial cells, unresponsiveness of vascular smooth muscle cells to NO during subarachnoid hemorrhage, and biased activation of sGC in vascular smooth muscle cells during acute exposure to hypoxia. The main message of this essay is that absence, blunting, or reversal of endotheliumdependent relaxations in response to vasodilator agonists cannot necessarily be interpreted as a sign of endothelial dysfunction. (This is a review article based on the invited lecture of the 58th Annual Meeting of Japanese College of Angiology.)

Keywords: endothelium, endothelial nitric oxide synthase, nitric oxide, soluble guanylyl cyclase, vascular smooth muscle

State Key Laboratory of Pharmaceutical Biotechnology and Department of Pharmacology and Pharmacy, Li Ka Shing Faculty of Medicine, The University of Hong Kong, Hong Kong, HKSAR, China

Received: December 18, 2017; Accepted: December 19, 2017 Corresponding author: Paul M. Vanhoutte, MD, PhD. State Key Laboratory of Pharmaceutical Biotechnology and Department of Pharmacology and Pharmacy, Li Ka Shing Faculty of Medicine, The University of Hong Kong, 21 Sassoon Road, Pokfulam, Hong Kong, HKSAR, China

Tel: + 852-9049-2727, Fax: +852-2817-0859

E-mail: vanhoutt@hku.hk

This is a review article based on the invited lecture of the 58th Annual Meeting of Japanese College of Angiology.

(cc) BY-NC-SA (02018 The Editorial Committee of Annals of Vascular Diseases. This article is distributed under the terms of the Creative Commons Attribution License, which permits use, distribution, and reproduction in any medium, provided the credit of the original work, a link to the license, and indication of any change are properly given, and the original work is not used for commercial purposes. Remixed or transformed contributions must be distributed under the same license as the original.

\section{Introduction}

When Professor Kimihiro Komori, the organizer of the 58th Annual Meeting of the Japanese College of Angiology, invited me to deliver a lecture and write this essay, obviously, I was flattered and accepted with pleasure, as he has been an important collaborator during my years at the Mayo Clinic in Rochester (MN, USA) and afterwards became a long-time friend. I intuitively knew that I would be expected to summarize the important protective role of endothelial cells in controlling local vasomotor control in health and vascular disease, since this has been the major interest of my group(s) during the last four decades. Central to this protective role of endothelial cells is endothelial nitric oxide synthase (eNOS; Fig. 1), when activated, generates nitric oxide (NO) besides other endotheliumderived relaxing factors (EDRFs; e.g., prostacyclin, hydrogen peroxide) and signals (e.g., ionic changes that evoke endothelium-dependent hyperpolarization [EDH]) that modulate responsiveness of the underlying vascular smooth muscle cells. When endothelial cells become dysfunctional with aging, obesity, and/or disease (e.g., diabetes and hypertension), the protective effect of NO is attenuated, which sets the stage for initiation of vasospasm and emergence of atherosclerosis. ${ }^{1-6)}$ When preparing my lecture, and thus this essay, I selected to address a misinterpretation often made from in vivo observations of endothelial function and dysfunction, particularly in humans where proper analysis is difficult. Indeed, too frequently, abnormal responses to endothelium-dependent vasodilators, in particular those to the gold-standard acetylcholine, ${ }^{7)}$ are viewed as an indicator solely of dysfunctional NO release. Although this clearly can be the case, abnormal responses to endothelium-dependent vasodilators can also be due to either unresponsiveness of the vascular smooth muscle to the relaxing effect of $\mathrm{NO}$ or to the paradoxical contractions initiated by the endothelial mediator. Examples, based on own experience, of those three possible reasons for apparent endothelial dysfunction are presented below.

\section{Insufficient NO}

The essential role of eNOS as a source of NO and its regulation (Fig. 1) as well as the impact of the endothe- 

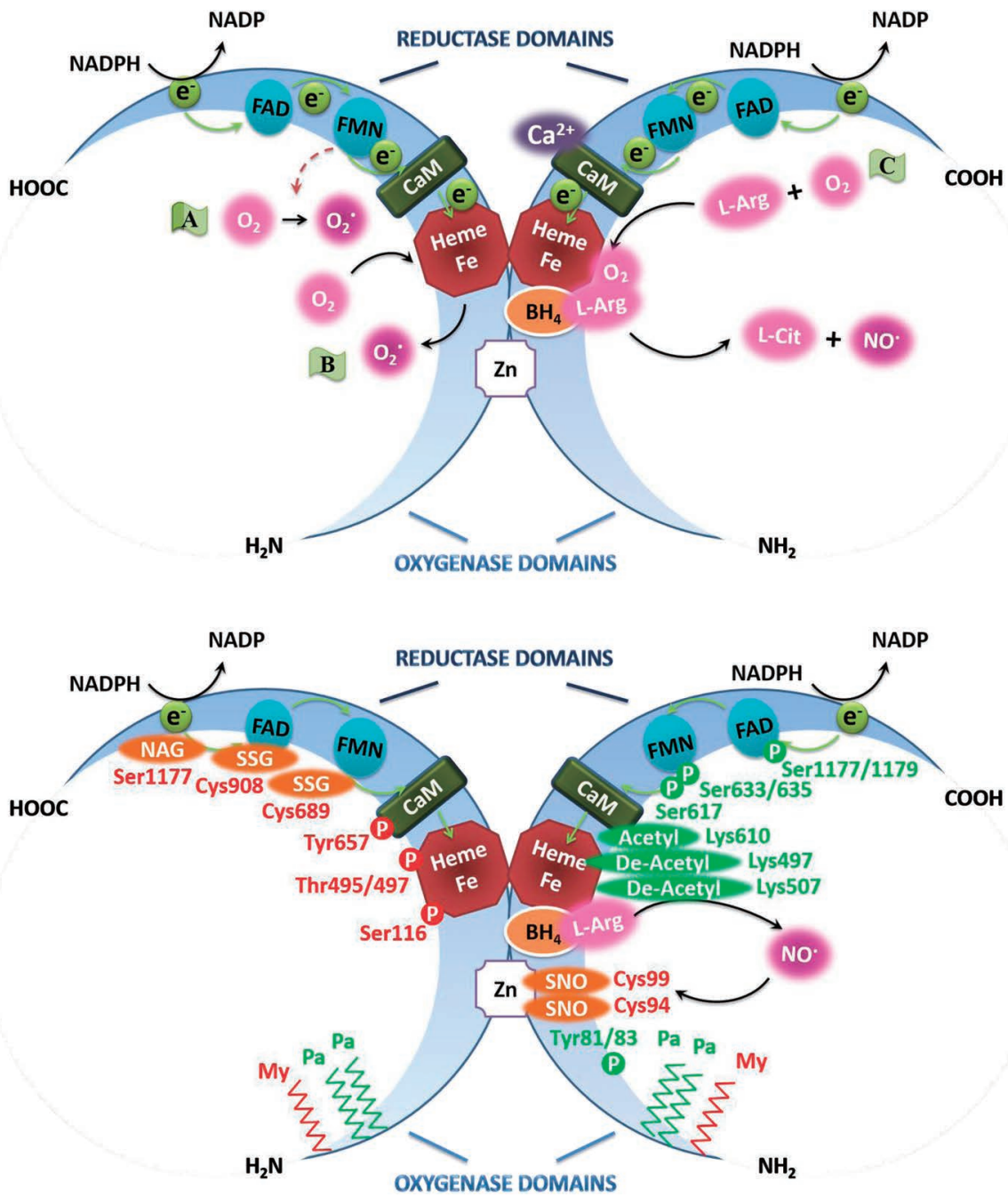

Fig. 1 Endothelial nitric oxide synthase. Upper: Production of nitric oxide (NO) by endothelial NO synthase (eNOS) requires not only the precursor L-arginine (L-Arg) but also the co-factors tetrahydrobiopterin $\left(\mathrm{BH}_{4}\right)$, flavin adenine dinucleotide (FAD), flavin mononucleotide (FMN), calmodulin (CaM), and iron protoporphyrin IX (Heme Fe). Although synthesized as monomers, eNOS has to form homodimers to produce NO. (A) As a monomer, eNOS produces superoxide anions $\left(\mathrm{O}_{2} \cdot{ }^{\cdot}\right)$ instead of $\mathrm{NO} \cdot(\mathrm{B})$ Even in the presence of eNOS homodimers, significant $\mathrm{O}_{2}$. production will occur when the effective concentrations of L-Arg is reduced below those required to saturate the enzyme. (C) When sufficient L-Arg and $\mathrm{BH}_{4}$ bind to coupled NOS, the electron (e-) flux provided by nicotinamide adenine dinucleotide phosphate (NADPH) passes through FAD, $\mathrm{FMN}$, CaM, and Heme Fe and is eventually used in $\mathrm{NO}$ production. $\mathrm{BH}_{4}$ is essential for eNOS coupling. Activity of the enzyme is increased when calcium ions $\left(\mathrm{Ca}^{2+}\right)$ bind to calmodulin. Lower: Principal post-translational modifications that enhance or reduce enzymatic activity and thus favor or reduce endothelium-dependent relaxations, respectively, are indicated in green and red, respectively. Cyst: cysteine; My: $\mathrm{N}$-myristoylation (at glycine 2); NAG: N-acetyl glucosamine; P: phosphorylation; Pa: thiopalmitoylation (at cysteines 15 and 26); Thr: threonine; Tyr: tyrosine; Ser: serine; SNO: S-nitrosylation; SSG: S-glutathionylation (Reproduced from Reference 5 by permission of the American Heart Association.)

lial mediator on its main target, soluble guanylyl cyclase (sGC) in underlying vascular smooth muscle cells (Fig. 2) have been reviewed extensively elsewhere. ${ }^{5,6)}$ Blood flowing through arteries and veins is an obvious source of signals that affect the endothelial cells, due not only to the shear stress that blood flow exerts but also the vasoactive substances such as catecholamines, vasopressin, insulin, and adiponectin that are contained within blood (Fig. 2). 


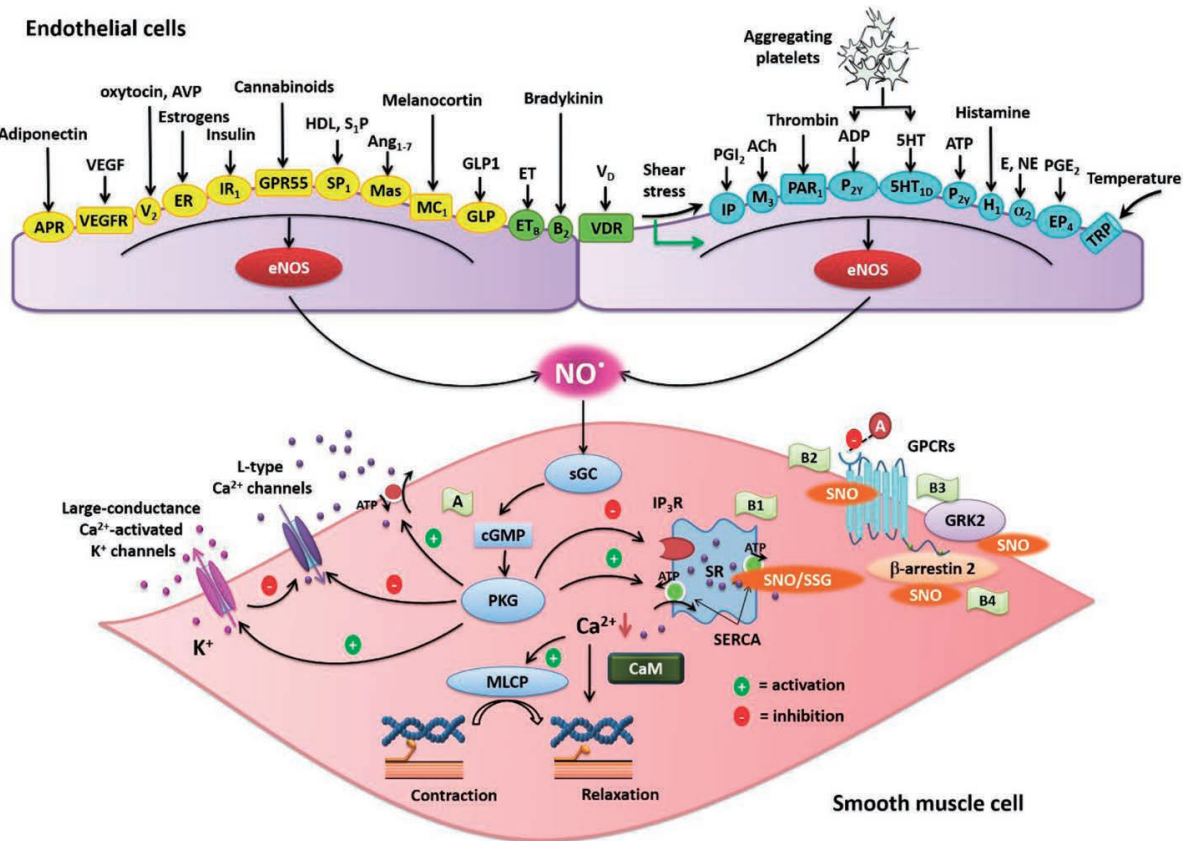

Fig. 2 Regulation of vascular tone by endothelium-derived nitric oxide. Upper: Physical factors, such as increases in shear stress and reduction in temperature, and neurohumoral mediators through the activation of specific endothelial cell membrane receptors cause instantaneous increases in the release of endothelium-derived nitric oxide by endothelial nitric oxide synthase (eNOS). Color code: Signals leading to eNOS activation that are dependent primarily on increases in calcium ion $\left(\mathrm{Ca}^{2+}\right)$ concentrations, post-translational modifications, or both are indicated by blue, yellow, and green, respectively. $\alpha$ : alpha adrenergic receptor; ACh: acetylcholine; ADP: adenosine diphosphate; Ang $_{1-7}$ : angioten$\sin _{1-7}$; AdipoR: adiponectin receptor; AVP: arginine vasopressin; B: bradykinin receptor; $\mathrm{E}$ : epinephrine; $\mathrm{EP}_{4}$ : prostaglandin $\mathrm{E}_{2}$-receptor 4; $\mathrm{ER}$ : non-genomic estrogen receptor; ET: endothelin-1, endothelin-receptor; GLP: glucagon-like peptide 1, glucagon-like peptide receptor; GPR55: G-protein coupled receptor 55; H: histaminergic receptor; HDL: high-density lipoprotein; 5HT: serotonin (5-hydroxytryptamine), serotoninergic receptor; IP: prostacyclin receptor; $\mathbb{I R}_{1}$ : insulin receptor; M: muscarinic receptor; Mas: Mas receptor; MC: melanocortin receptor; NE: norepinephrine; $\mathrm{P}$ : purinergic receptor; PAR: protease-activated receptor; $\mathrm{PGE}_{2}$ : prostaglandin $\mathrm{E}_{2} ; \mathrm{PGI}_{2}$ : prostacyclin; $\mathrm{SP}_{1}$ : sphingosine 1-phosphate; TRP: transient potential receptor V4; V: vasopressin receptor; VEGF: vascular endothelial growth factor; VEGFR: VEGF-receptor; $V_{D}$ : vitamin D; VDR: vitamin $\mathrm{D}$ receptor. Lower: Contraction of vascular smooth muscle cells is initiated by increases in intracellular calcium $\left(\mathrm{Ca}^{2+}\right)$ concentration either from internal stores (sarcoplasmic reticulum, SR) or by influx into the cell following opening of calcium channels in the cell membrane. The intracellular free calcium ions bind to calmodulin (CaM), and the calcium-calmodulin complexes activate myosin light-chain kinase, which phosphorylates myosin light chain that leads to cross-bridge formation between the myosin heads and actin filaments. Cross-bridge formation results in contraction of the smooth muscle cell. NO modulates the contraction process in different ways. (A) NO stimulates soluble guanylyl cyclase (sGC) in vascular smooth muscle cells, which produces 3 '-5'-cyclic guanosine monophosphate (cGMP). Cyclic GMP activates protein kinase $\mathrm{G}(\mathrm{PKG})$, preventing $\mathrm{Ca}^{2+}$ influx through voltage-dependent calcium channels and calcium release mediated by inositol 1,4,5-trisphosphate $\left(\mathrm{IP}_{3}\right)$ receptors $\left(\mathrm{IP}_{3} \mathrm{R}\right)$. In addition, $\mathrm{PKG}$ not only activates the calcium ATPase on the cell membrane and the sarco/endoplasmic reticulum calciumATPase (SERCA) to accelerate the efflux of cytosolic $\mathrm{Ca}^{2+}$ to the outside the cell and its reuptake into the SR, respectively, but also opens large-conductance calcium-activated potassium channels, resulting in hyperpolarization which in turn prevents the opening of voltage-dependent L-type channels, hence reducing the $\mathrm{Ca}^{2+}$ influx. The resulting decrease in free intracellular $\mathrm{Ca}^{2+}$ concentrations inactivates calmodulin and thus myosin light chain kinase. Calcium depletion also augments the activity of myosin light-chain phosphatase (MLCP). The actin-myosin cross-bridges are interrupted, and the vascular smooth muscle cell relaxes. (B) NO also S-nitrosylates (SNO) the following intracellular proteins: 1) SERCA, which results in accelerated $\mathrm{Ca}^{2+}$ depletion; $\mathrm{NO}$ also facilitates the S-glutathionylation (SSG) of SERCA; 2) G protein-coupled receptors (GPCRs), which results in reduced binding of ligands (A) or blunted G-protein coupling; 3) G proteincoupled receptor kinase 2 (GRK2), which prevents desensitization and internalization of $\beta$-adrenoceptors; and 4) $\beta$-arrestin 2 , which increases receptor internalization. (Reproduced from Reference 5 by permission of the American Heart Association.) 

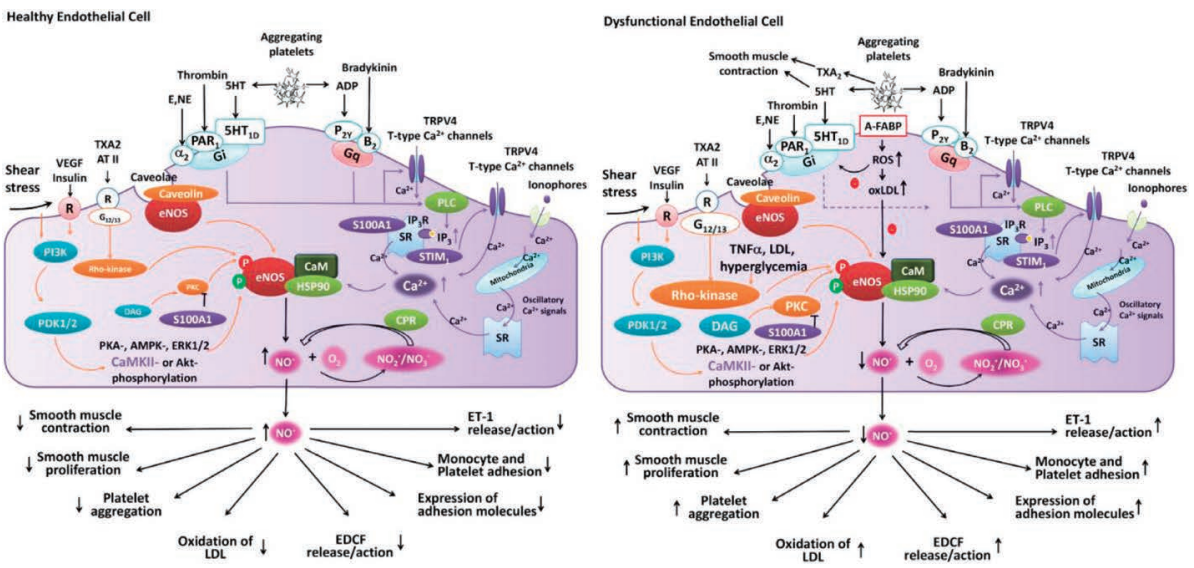

Fig. 3 Production of nitric oxide by endothelial cells. Left: Healthy endothelial cells. Endothelial nitric oxide synthase (eNOS) can be activated in calcium-dependent or calcium-independent ways. Conversely, agonists (e.g., acetylcholine, adenine nucleotides, bradykinin, catecholamines, endothelin-1, histamine, serotonin, and thrombin) bind to specific receptors or open ion channels on the endothelial cell membrane to increase the influx of calcium $\left(\mathrm{Ca}^{2+}\right)$ and/or to induce $\mathrm{Ca}^{2+}$ release from intracellular stores (e.g., by stimulating the formation of inositol triphosphate $\left[\mathrm{IP}_{3}\right]$ ), in particular the sarcoplasmic reticulum (SR) and mitochondria. The calcium ions bind to calmodulin (CaM), which leads to the activation of the CaM-binding domain of eNOS to produce nitric oxide (NO). Association of the calmodulin-eNOS complex with heat shock protein 90 (HSP 90) increases enzyme activity; increases in intracellular $\mathrm{Ca}^{2+}$ concentration caused by ionophores such as A23187 also augment the production of NO by eNOS. Conversely, increases in hemodynamic shear stress and binding of agonists such as certain hormones (adiponectin, insulin) or growth factors (vascular endothelial growth factor) to their specific receptors initiate phosphorylation $(P)$ of eNOS through activation of the phosphoinositide 3-kinase (PI3K)-phosphoinositidedependent kinase-1/2 (PDK1/2) pathway. This event stimulates kinases (protein kinase A [PKA], AMP-activated protein kinase [AMPK], $\mathrm{Ca}^{2+} /$ calmodulin dependent protein kinase II [CaMKII], extracellular signal-regulated kinases 1/2 [ERK1/2] and protein kinase B [Akt]). Phosphorylation events that enhance or reduce enzymatic activity and thus favor or reduce endothelium-dependent relaxations, respectively, are indicated in green and red, respectively. Endothelial cell membrane receptors are coupled to the activation of eNOS through different $\mathrm{G}$ proteins ( $\mathrm{Gi}$ and $\mathrm{Gq}$, inhibited or not, respectively, by pertussis toxin). In addition to eNOS, NO can be produced from nitrate by cytochrome $\mathrm{P} 450$ reductase (CPR). Once released, NO exerts a protective effect beyond its direct control of vascular tone by inhibiting (downward arrows) a number of processes favoring vasospasm and initiation of atherosclerosis. Right: Dysfunctional endothelial cells. In regenerated endothelial cells, the overexpression of fatty acid-binding protein $A(A-F A B P)$ leads to increased production of reactive oxygen species (ROS), favoring the formation of oxidized low-density lipoproteins (oxLDL), which interrupts (-) the Gi-mediated activation of eNOS. In addition, if Rho-kinase and protein kinase $\mathrm{C}(\mathrm{PKC})$ are activated, the ensuing phosphorylation events reduce eNOS activity. As a consequence, a number of processes are disinhibited (upward arrows), which favor the initiation of vasospasm and atherosclerosis. $\alpha$ : alpha adrenergic receptor; ADP: adenosine diphosphate; AT II: angiotensin II; B: bradykinin receptor; DAG: diacylglycerol; E: epinephrine (adrenaline); ET-1: endothelin-1; EDCFs: endothelium-derived vasoconstrictor prostanoids; $5 \mathrm{HT}$ : serotonin (5-hydroxytryptamine); G: coupling proteins; LDL: low-density lipoprotein; NE: norepinephrine (noradrenaline); $\mathrm{P}$ : purinoceptor; PAR: protease-activated receptor; R: receptor; TRP: transient potential receptor; TX: thromboxane; S100A1: protein S100A1; STIM : SR-transmembrane protein stromal interaction molecule 1; VEGF: vascular endothelial growth factor (Reproduced from Reference 5 by permission of the American Heart Association.) 
A

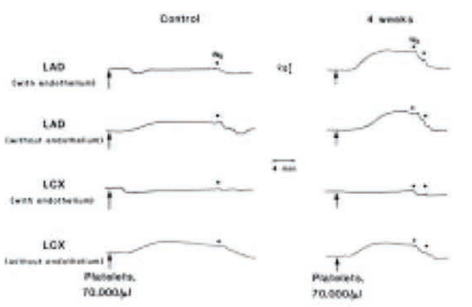

C

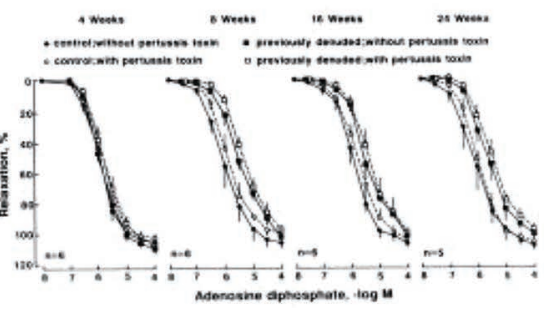

B

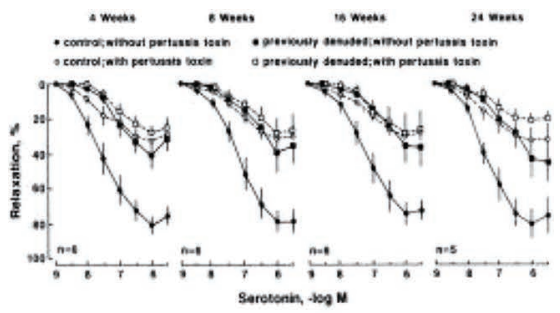

D

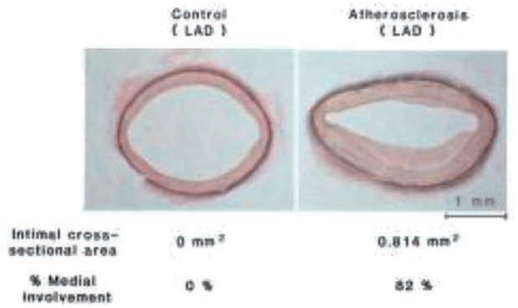

Fig. 4 Selective dysfunction of regenerated endothelium. Upper (A) Platelets aggregating in the vicinity of quiescent isolated rings of porcine left anterior descending (LAD) and left circumflex (LCX) coronary arteries cause minimal changes in isometric tension if the native (control) endothelium is present but lead to contractions if the endothelium has been removed. In contrast, if the LAD rings are lined with regenerated endothelial cells, the aggregating platelets cause vigorous contractions four weeks after angioplasty, which are not observed in LCX preparations of the same heart covered with native endothelial cells (Data from Reference 12). Upper (B) Relaxations in response to increasing concentrations of serotonin in precontracted rings of porcine LAD rings lined with either native (control) or regenerated (previously denuded) endothelium, in the absence and presence of the $\mathrm{G}_{\mathrm{i}}$-protein inhibitor pertussis toxin; the relaxations are considerably reduced in the latter preparations, and pertussis toxin inhibits response only in arteries covered with native endothelium. These experiments demonstrate that $\mathrm{G}_{\mathrm{i}}$-protein coupling is dysfunctional in regenerated endothelial cells, which persists for at least six months after the denudation procedure (Data from Reference 12). Lower (C) Relaxations in response to increasing concentrations of adenosine diphosphate in precontracted rings of porcine LAD rings lined with either native (control) or regenerated (previously denuded) endothelium, in the absence and presence of the $\mathrm{G}_{\mathrm{i}}$-protein inhibitor pertussis toxin; the relaxations are comparable between the two groups, irrespective of the time after denudation, and pertussis toxin does not inhibit the response (Data from Reference 12). These experiments demonstrate that $\mathrm{G}_{\mathrm{q}}$-protein coupling is functional in regenerated endothelial cells. Lower (D) Morphology of LAD rings covered with regenerated endothelium of pigs fed for two months with either control (left) or high-fat (right) diet after angioplastic denudation. Typical atherosclerotic lesions are observed in preparations from an animal receiving the high-fat diet, but such lesions are seen, although not shown here, only in the previously denuded part of the coronary artery (data from Reference 17).

Among the latter, products released by aggregating platelets (serotonin [5-hydroxytryptamine, 5-HT], adenosine triphosphate [ATP], and adenosine diphosphate [ADP]) or formed during blood coagulation (thrombin) evoke endothelium-dependent relaxations. ${ }^{5,6)}$ Thus, when platelets aggregate in vitro in the vicinity of endothelial cells, they evoke such relaxations of isolated animal and human arteries and veins, by virtue of their ability to release serotonin and ADP in amounts that are sufficient to activate eNOS $^{8-14)}$ (Fig. 3). The endothelial cell membrane 5-HT $1 \mathrm{D}$ responding to serotonin released by aggregating platelets are coupled to eNOS by $G_{i}$ proteins, whereas the $P_{2 y}$ pu- rinoceptors activated by ADP as well as ATP are linked to the enzyme by $G_{\mathrm{q}}$ proteins ${ }^{12,13,15)}$ (Fig. 3, Left). When the endothelial lining is absent, such as that occurs following injury, the vasoconstrictors serotonin and thromboxane $\mathrm{A}_{2}$ released by the aggregating platelets reach vascular smooth muscle cells and lead to their contraction, initiating the vascular phase of hemostasis. NO-mediated responses to aggregating platelets are facilitated by chronic intake of $\omega-3$ polyunsaturated fatty acids but are reduced with high-fat/high-cholesterol diets. ${ }^{16-19)}$

In mature arteries and veins, endothelial cells form a tight monolayer and remain quiescent for years. I remem- 


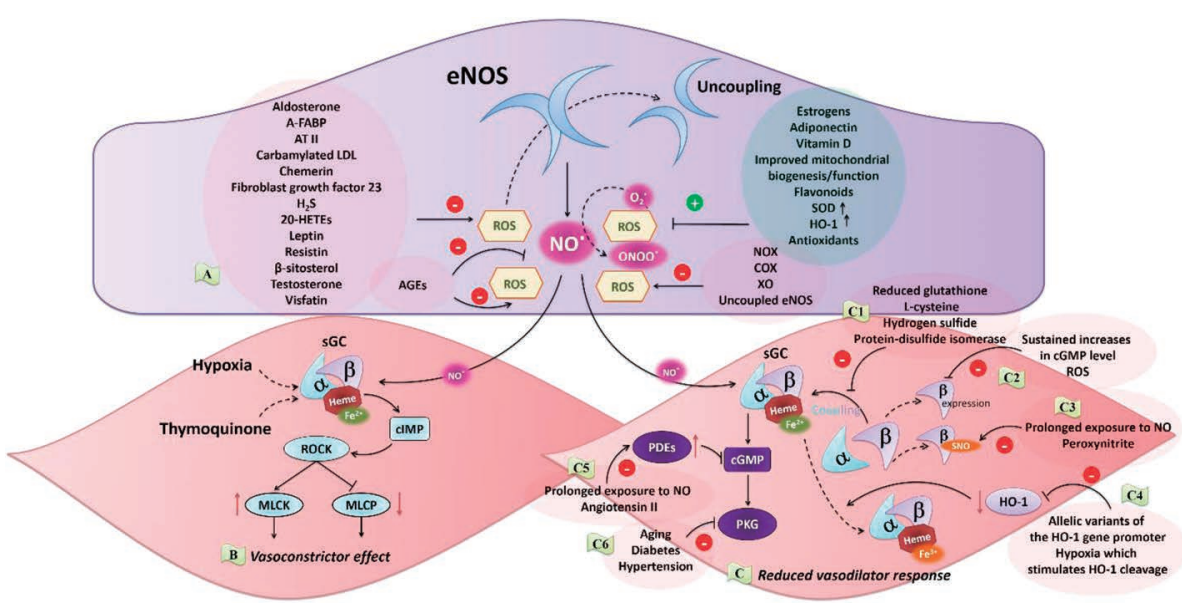

Fig. 5 Apparent endothelial dysfunction. Upper (A) Examples of factors which reduce or enhance the production of reactive oxygen species (ROS; superoxide anions in particular) and thus favor or inhibit nitric oxide (NO)-mediated, endothelium-dependent relaxation are indicated by green $(+)$ and red $(-)$ symbols, respectively. NO scavenging by superoxide anions that lead to the formation of peroxynitrite can occur in endothelial cells, intercellular space, or vascular smooth muscle cells. In addition to scavenging NO, ROS also uncouple eNOS. Lower: Examples of factors that alter the responsiveness of vascular smooth muscle soluble guanylyl cyclase (sGC) to endothelium-derived NO. Lower, left (B) Under hypoxic conditions or upon exposure to thymoquinone, the biased activity of SGC produces $3^{\prime}-5$ '-cyclic inosine monophosphate (cIMP) instead of 3'-5'-cyclic guanosine monophosphate (cGMP), which activates Rho-associated protein kinase (ROCK) and inhibits myosin light-chain phosphatase (MLCP), facilitating contraction. Lower, right (C) Reduced activity of sGC can be due to: $\mathrm{C} 1$ ) reduced dimerization by thiol-reducing agents, $\mathrm{C} 2$ ) reduced expression of the $\beta$-subunit, C3) desensitization by $S$-nitrosylation at Cyst122 of the $\beta$-subunit, and $\mathrm{C} 4$ ) reduced expression/activity of heme oxygenase (HO)-1, favoring oxidation of the sGC-heme (containing $\mathrm{Fe}^{3+}$ instead of $\mathrm{Fe}^{2+}$ ). Reduced vascular responses to endothelium-derived NO can be due to increased expression/presence of phosphodiesterases (PDEs), which reduce cGMP (C5) or reduced expression/activity of protein kinase $G(P K G)(C 6)$. $\alpha, \beta$ :subunits of sGC; AGE: advanced glycation end-products; AT II: angiotensin II; COX: cyclooxygenase; eNOS: endothelial nitric oxide synthase; $\mathrm{H}_{2} \mathrm{~S}$ : hydrogen sulfide; LDL: low-density lipoprotein; MLCK: myosin light-chain kinase; NOX: NADPH oxidase; SNO: S-nitrosylation; SOD: superoxide dismutase; 20-HETE: 20-hydroxyeicosatetraenoic acid; XO: xanthine oxidase; downward arrows: -, inhibition; upward arrows: +, stimulation (Reproduced from Reference 5 by permission of the American Heart Association.)

ber reading a report of autopsies on young healthy adults, all victims of road accidents, stressing the point that signs of endothelial cell turnover were observed only in people older than thirty years of age; unfortunately, I lost track of this reference and thus cannot share it with the readers. However, sooner or later, apoptotic programming initiates detachments of endothelial cells, which are removed by blood flow. This turnover is most prominent in areas of the vascular tree exposed to higher shear stress and is accelerated by aging, exposure to chronic increases in arterial pressure (i.e., hypertension), and prolonged oxidative stress such as that occurs in diabetes. Disruption of the endothelial lining is also unavoidable during acute cardiological procedures, angioplasty in particular, ${ }^{12,13)}$ heart transplantation, ${ }^{20,21)}$ and venous grafting. ${ }^{22,23)}$ De- nuded/injured parts of the intima are rapidly covered by "regenerated" endothelial cells that originate from cells at the rims of injury or are derived from circulating endothelial progenitor cells. ${ }^{4-6)}$ However, these regenerated endothelial cells are a prototypical example of selective endothelial dysfunction due to decreased production of $\mathrm{NO}$ in response to aggregating platelets. This conclusion is based on repeated observations in porcine coronary circulation. When relining of the endothelial surface was complete four weeks after in vivo angioplasty, preparations covered with regenerated endothelium were characterized by a reduction in relaxation in response to aggregating platelets, serotonin, $\alpha_{2}$-adrenergic agonists, and thrombin, whereas their response to ADP or bradykinin remained unchanged $^{4-6,12-14)}$ (Fig. 4). These findings prompted the 
$\mathbf{A}$

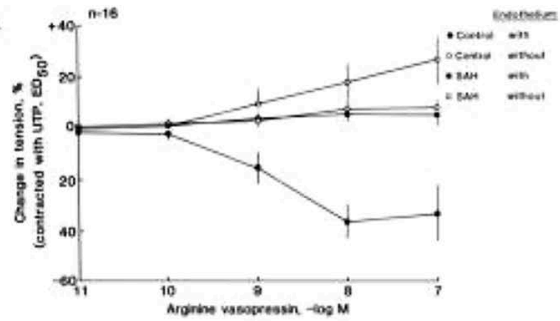

C

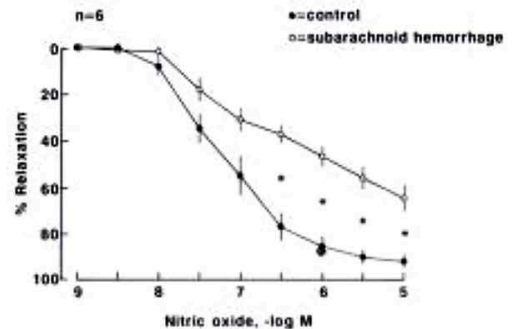

B

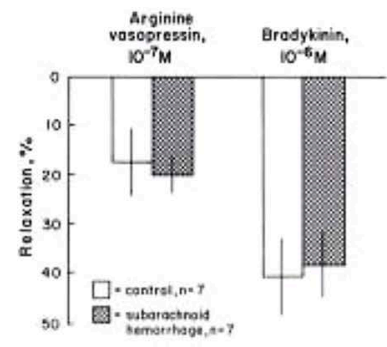

D

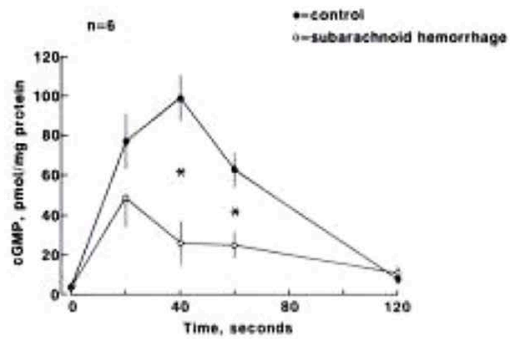

Fig. 6 Apparent endothelial dysfunction in canine basilar arteries after subarachnoid hemorrhage (SAH), mimicked by injection of autologous blood in cisterna magna. Upper (A) Arginine vasopressin causes concentration-dependent relaxations in control arteries with endothelium but not in those without endothelium; such relaxations are not observed in preparations exposed to SAH in vivo one week earlier (Data from Reference 25). Upper (B) Bioassay of endothelium-derived nitric oxide (NO) during relaxations of control canine basilar arteries and preparations exposed to $\mathrm{SAH}$ earlier in vivo, in response to either arginine vasopressin or bradykinin; the release of the endothelium-derived mediator is comparable between the two groups (Data from Reference 26). Lower (C) Relaxations in rings with endothelium of control canine basilar arteries and preparations subjected to SAH in vivo earlier, in response to increasing concentrations of authentic NO; the relaxations are reduced in the latter (Data from Reference 27). Lower (D) Production of 3'-5'-cyclic guanosine monophosphate (cGMP) of rings with endothelium of control canine basilar arteries and preparations subjected to $\mathrm{SAH}$ in vivo earlier, in response to bradykinin $\left(10^{-7} \mathrm{M}\right)$; the production of the second messenger is reduced in the latter (Data from Reference 27). These experiments demonstrate that the reduced endothelium-dependent relaxations (Upper

[A]) are not due to a dysfunction in NO release by the endothelial cells.

concept that selective dysfunction of $\mathrm{G}_{\mathrm{i}}$-related, endothelium-dependent relaxations are an unavoidable consequence of the regenerative process (Fig. 3, Right) ${ }^{4-6)}$ and a characteristic of regenerated endothelial cells. In vitro, this selective endothelial dysfunction results in exaggerated vasoconstrictor responses to serotonergic activation by aggregating platelets (Fig. 4A) and serotonin. In vivo, this long-lasting dysfunction favors hyperconstriction, i.e., vasospasm, in response to aggregating platelets or exogenous serotoninergic agonists and favors the formation of atherosclerotic lesions ${ }^{46,24)}$ (Fig. 4D). The molecular mechanisms underlying the selective loss of $\mathrm{G}_{\mathrm{i}}$-dependent responses (Figs. 4B and 4C) have been summarized in detail elsewhere. ${ }^{4-6,24)}$ Nonetheless, these earlier experiments caution against categorizing relaxations/dilatations in response to a single endothelium-dependent agonist as endothelial dysfunction. This is in particular important in the case of acetylcholine which, in many blood vessels, is a full, powerful stimulator of eNOS and thus less likely to be modulated, whereas it simply does not evoke endothe- lium-dependent, NO-mediated relaxations in other vessels such as porcine and human coronary arteries, and canine basilar arteries. ${ }^{3)}$

\section{Insensitivity of Vascular Smooth Muscle despite Sufficient NO Release}

Under a number of conditions such as aging, diabetes, hypertension, or prolonged exposure to exogenous $\mathrm{NO}$ donors or angiotensin II (Fig. 5, Lower right), endothelial cells do indeed release NO upon exposure to endotheliumdependent relaxing agents; however, the underlying vascular smooth muscle has become partially or completely insensitive to the vasodilator effect of the mediator..$^{5)}$ The most convincing example of this situation comes from our group when, many years ago, we investigated the impact of subarachnoid hemorrhage on endothelium-dependent relaxations in the canine basilar artery. ${ }^{25-27)}$ In those studies, angiographically confirmed vasospasm of the basilar artery was present seven days after the injection of 
autologous blood in cisterna magna. Isolated rings of the vasospastic basilar arteries did not relax when exposed to increasing concentrations of vasopressin, unlike the powerful endothelium-dependent vasodilator acetylcholine, or ADP (Fig. 6A). When we perfused preparations to bioassay NO release, we were quite surprised to observe that the release of endothelium-derived relaxing factors by the vasospastic arteries was comparable to that of healthy controls (Fig. 6B). Next, we demonstrated that the vascular smooth muscle cells of the vasospastic arteries were indeed significantly less sensitive to authentic NO than control preparations (Fig. 6C). Finally, we explained this insensitivity by a reduction in the production of $3^{\prime}, 5^{\prime}$-cyclic guanosine monophosphate (cGMP), the second messenger formed by the enzyme sGC, which, as noted earlier, is the main target for $\mathrm{NO}$ in vascular smooth muscle cells (Fig. 6D). Thus, the unavoidable conclusion was that subarachnoid hemorrhage did not cause endothelial dysfunction, since the endothelial cells fulfill their function quite well to release sufficient NO; rather, it prevented the endothelium-derived mediator from inducing the relaxation of the underlying vascular smooth muscle cells. In hindsight, this conclusion is not too surprising since, with the formation of the autologous blood clot at the outside of the basilar artery, hemoglobin released by lysed erythrocytes can diffuse to the vascular smooth
A

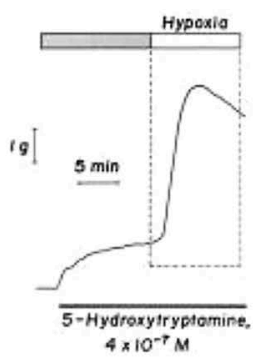

C

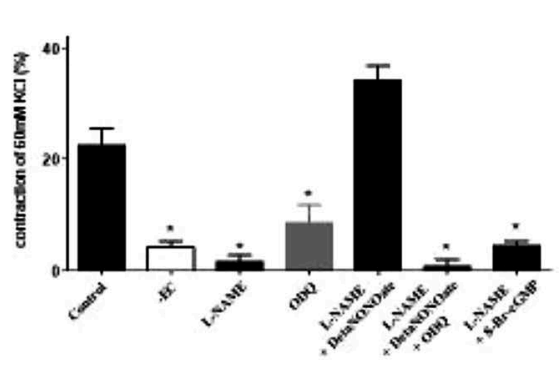

B

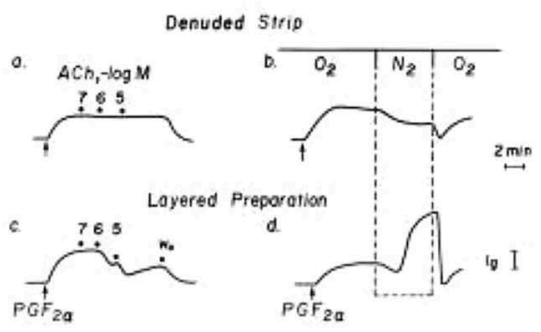

D

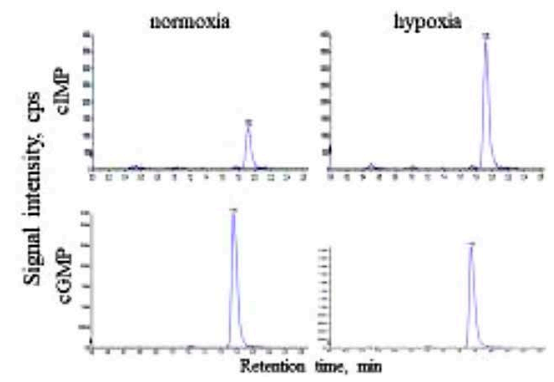

Fig. 7 Endothelium-dependent contractions in response to hypoxia. Upper (A) Augmentation of the contraction of a canine coronary artery ring to serotonin (5-hydroxytryptamine) by acute hypoxia (Data from Reference 31). Upper (B) Experiments under bioassay conditions. If a layered (i.e., sandwich [7]) preparation contains no endothelium (upper), acetylcholine (ACh) evokes no response during a contraction by prostaglan$\operatorname{din} \mathrm{F}_{2 \alpha}\left(\mathrm{PGF}_{2 \alpha}\right)$, while acute hypoxia causes relaxation. However, if the layered preparation contains endothelial cells (lower), ACh causes a concentration-dependent relaxation while acute hypoxia evokes an increase in tension (Data from Reference 34). These experiments demonstrate that the signal leading to hypoxic augmentation originates in endothelial cells. Lower (C) Hypoxic augmentation in rings of porcine coronary arteries. The augmentation observed in rings with endothelium under control conditions (Control) is reduced/abolished by endothelium-removal (-EC), by an eNOS-inhibitor (L-NAME) or by an SGC-inhibitor (ODQ). In the presence of L-NAME, the exogenous NO donor detaNONOate reinstalls hypoxic augmentation, an effect which is abrogated by ODQ. In contrast, in the presence of the eNOS-inhibitor, the cell permeable 3',5'-cyclic guanosine monophosphate (cGMP) analog 8-Br-cGMP does not restore the hypoxic augmentation (Data from Reference 40). These experiments demonstrate that the release of $\mathrm{NO}$ and the activation of $\mathrm{SGC}$, but not the production of cGMP, underlie endothelium-dependent contractions in response to acute hypoxia. Lower (D) Production of 3',5'-cyclic inosine monophosphate (cIMP, upper) and cGMP (lower) by porcine coronary arteries under normoxic (left) and hypoxic (right) conditions (Data from Reference 42). These experiments demonstrate that hypoxia augments the release of the non-canonical sGC-product cIMP, while, if anything, decreasing that of $c G M P$. 
muscle cells and interfere with the enzymatic activity of sGC in these cells. In addition, caution is warranted in analysis of the impact of adventitial events on endothelial function per se. For example, it is not always easy to demonstrate that inflammatory responses in periadventitial adipose tissue (PVAT) and the release of adipokines from that site ${ }^{28,29)}$ truly affect the release of NO by endothelial cells. In particular, in larger arteries with a thick media, these adipokines have a long way to go before they can reach the endothelium and alter the responsiveness of vascular smooth muscle cells that they encounter first. In any case, I never accept conclusions concerning endothelial dysfunction if reduced relaxations (in vitro) or dilatations (in vivo) to endothelium-dependent vasodilators are not paralleled by data demonstrating unchanged responses to exogenous NO donors.

\section{Altered Responsiveness of Vascular Smooth Muscle despite Sufficient NO Release}

The prototypical example of a situation where NO released by endothelial cells results in vasoconstriction due to altered responsiveness of the underlying vascular smooth muscle is endothelium-dependent contractions in response to hypoxia. For almost half a century, I have been puzzled by the observation that acute hypoxia, which I considered to be the ultimate vasodilator signal as a physiologist, in fact can cause temporary contractions of certain isolated blood vessels ${ }^{30,31)}$ (Fig. 7A). A breakthrough came when we discovered that such responses required the presence of endothelial cells in the isolated preparations studied. ${ }^{32,33)}$ The next step was the demonstration that the release of an endothelium-derived factor was indeed pivotal for evoking hypoxic augmentation of vasoconstrictor responses under bioassay conditions ${ }^{34)}$ (Fig. 7B). Contractions of isolated blood vessels in response to the release of endothelium-derived contracting factors (EDCFs; mainly vasoconstrictor prostanoids and possibly endothelin-1) is well established, but such responses are exacerbated in the absence of NO., ${ }^{2,35-37)}$ In contrast, a series of pharmacological experiments using inhibitors of both eNOS and sGC demonstrated the requirement of endothelium-derived $\mathrm{NO}$ and subsequent activation of sGC as prerequisites for hypoxic augmentation ${ }^{38-40)}$ (Fig. 7C). However, the hypoxic augmentation could not possibly be attributed to increased production of cGMP, the canonical product of sGC. ${ }^{40)}$ Revelation came when we found that sGC could switch from guanosine as a substrate (to produce cGMP) to inosine (to produce $3^{\prime}, 5^{\prime}$-cyclic inosine monophosphate [cIMP]) under certain circumstances. ${ }^{41)}$ Sure enough, when measuring the production of both cGMP and cIMP during hypoxic augmentations, it became apparent that the levels of the former, if anything, were reduced, whereas those of the latter were increased significantly (Fig. 7D ${ }^{42)}$; the obvious counterintuitive conclusion of these experiments was that the non-canonical sGC-product cIMP was responsible for the endothelium-dependent augmentations caused by acute exposure to hypoxia. An identical interpretation was reached when analyzing the endothelium-dependent augmentations observed with the natural product thymoquinone. ${ }^{43}$ These experiments lead to the conclusion that hypoxia and certain quinones biased the activity of sGC in vascular smooth muscle cells (Fig. 5, Lower left), although it was not easy for our interpretation to bypass the criticism of reviewers. ${ }^{5,44-46)}$ We are currently investigating intracellular target(s) of hypoxia and quinones that cause the biased activity of sGC, with a particular interest in the possible role of the enzyme $\mathrm{NAD}(\mathrm{P}) \mathrm{H}$ : quinone acceptor oxidoreductase-1 (NQO-1). Nonetheless, the epic mission to uncover the mechanism underlying endothelium-dependent contractions to acute hypoxia is probably the best example in a long career that one should never forget the odd, unexplainable behavior of an isolated blood vessel ${ }^{30)}$ but should revisit over the years as a satisfactory explanation becomes available with the progression of knowledge and technology. In the case of hypoxic augmentations, this is not only a matter of "science for the sake of science" as we were able to demonstrate earlier that in coronary circulation previous ischemia/reperfusion markedly exaggerates the development of hypoxic augmentations and thus facilitates hypoxic vasospasm, ${ }^{39)}$ an exaggeration which helps, at least us, to better understand why patients with sleep apnea exhibit a greater propensity for acute coronary syndrome.

\section{Conclusion}

The three examples summarized above yield the following lessons on possible alterations in the eNOS-NO-sGC pathway while being challenged with abnormal responses, i.e., relaxations (in vitro) or dilatations (in vivo), to accepted endothelium-dependent vasodilators: a) never trust the absence of differences if only one agonist is used, especially acetylcholine; b) always ensure that the responsiveness of the underlying vascular smooth muscle cells to NO is unchanged; and c) also consider that the unexpected responses, in particular endothelium-dependent contractions, can be due to biased responses of target(s) for NO in vascular smooth muscle cells.

\section{Acknowledgments}

The author thanks Mrs. Ivy Wong sincerely for her usual, most efficient editorial assistance and Dr. Susan Leung for help with rearranging the figures. He also thanks his 
former and current collaborators for their inspiration during the studies briefly summarized in this essay, with particular gratitude to a group of outstanding Japanese post-doctoral fellows: Kimihiro Komori, Hiroaki Shimokawa, and Phyo Kim.

\section{Disclosure Statement}

No conflicts of interest are reported.

\section{References}

1) Furchgott RF, Vanhoutte PM. Endothelium-derived relaxing and contracting factors. FASEB J 1989; 3: 2007-18.

2) Vanhoutte PM. Endothelium-dependent contractions in hypertension: when prostacyclin becomes ugly. Hypertension 2011; 57: 526-31.

3) Vanhoutte PM. Endothelial lessons. Curr Vasc Pharmacol 2016; 14: 175-80.

4) Vanhoutte PM. Regenerated endothelium and its senescent response to aggregating platelets. Circ J 2016; 80: 783-90.

5) Vanhoutte PM, Zhao Y, Xu A, et al. Thirty years of saying NO: sources, fate, actions and misfortunes of the endothelium-derived vasodilator mediator. Circ Res 2016; 119: 375 96.

6) Vanhoutte PM, Shimokawa H, Félétou M, et al. Endothelial dysfunction and vascular disease - a 30th anniversary update. Acta Physiol (Oxf) 2017; 219: 22-96.

7) Furchgott RF, Zawadzki JV. The obligatory role of endothelial cells in the relaxation of arterial smooth muscle by acetylcholine. Nature 1980; 288: 373-6.

8) Cohen RA, Shepherd JT, Vanhoutte PM. Inhibitory role of the endothelium in the response of isolated coronary arteries to platelets. Science 1983; 221: 273-4.

9) Cohen RA, Shepherd JT, Vanhoutte PM. Endothelium and asymmetrical responses of the coronary arterial wall. Am J Physiol 1984; 247: H403-8.

10) Houston DS, Shepherd JT, Vanhoutte PM. Aggregating human platelets cause direct contraction and endotheliumdependent relaxation of isolated canine coronary arteries. Role of serotonin, thromboxane A2, and adenine nucleotides. J Clin Invest 1986; 78: 539-44.

11) Komori K, Shimokawa H, Vanhoutte PM. Endotheliumdependent relaxation in response to aggregating platelets in porcine femoral veins and its modulation by diet. Circulation 1989; 80: 401-9.

12) Shimokawa H, Flavahan NA, Vanhoutte PM. Natural course of the impairment of endothelium-dependent relaxations after balloon endothelium removal in porcine coronary arteries. Possible dysfunction of a pertussis toxin-sensitive $G$ protein. Circ Res 1989; 65: 740-53.

13) Shimokawa H, Flavahan NA, Vanhoutte PM. Loss of endothelial pertussis toxin-sensitive G-protein function in atherosclerotic porcine coronary arteries. Circulation 1991; 83: 652-60.

14) Zellers TM, Shimokawa H, Yunginger J, et al. Heterogeneity of endothelium-dependent and endothelium-independent responses to aggregating platelets in porcine pulmonary arteries. Circ Res 1991; 68: 1437-45.

15) Flavahan NA, Shimokawa H, Vanhoutte PM. Pertussis toxin inhibits endothelium-dependent relaxations to certain agonists in porcine coronary arteries. J Physiol 1989; 408: 549-60.

16) Shimokawa H, Lam JY, Chesebro T, et al. Effects of dietary supplementation with cod-liver oil on endothelium-dependent responses in porcine coronary arteries. Circulation 1987; 76: 898-905.

17) Shimokawa H, Vanhoutte PM. Hypercholesterolemia causes generalized impairment of endothelium-dependent relaxation to aggregating platelets in porcine arteries. J Am Coll Cardiol 1989; 13: 1402-8.

18) Komori K, Shimokawa H, Vanhoutte PM. Hypercholesterolemia impairs endothelium-dependent relaxations to aggregating platelets in porcine iliac arteries. J Vasc Surg 1989; 10: 318-25.

19) Kim P, Shimokawa H, Vanhoutte PM. Dietary $\omega-3$ fatty acids and endothelium-dependent responses in porcine cerebral arteries. Stroke 1992; 23: 407-13.

20) Perrault LP, Bidouard JP, Janiak P, et al. Time course of coronary endothelial dysfunction in acute untreated rejection after heterotopic heart transplantation. J Heart Lung Transplant 1997; 16: 643-57.

21) Perrault LP, Bidouard JP, Janiak P, et al. Impairment of G-protein-mediated signal transduction in the porcine coronary endothelium during rejection after heart transplantation. Cardiovasc Res 1999; 43: 457-70.

22) Komori K, Gloviczki P, Bourchier RG, et al. Endotheliumdependent vasorelaxations in response to aggregating platelets are impaired in reversed vein grafts. J Vasc Surg 1990; 12: $139-47$.

23) Komori K, Schini VB, Gloviczki P, et al. The impairment of endothelium-dependent relaxations in reversed vein grafts is associated with a reduced production of cyclic guanosine monophosphate. J Vasc Surg 1991; 14: 67-75.

24) Chan CK, Zhao Y, Liao SY, et al. A-FABP and oxidative stress underlie the impairment of endothelium-dependent relaxations to serotonin and the intima-medial thickening in the porcine coronary artery with regenerated endothelium. ACS Chem Neurosci 2013; 4: 122-9.

25) Kim P, Sundt TM Jr, Vanhoutte PM. Alterations in endothelium-dependent responsiveness of the canine basilar artery after subarachnoid hemorrhage. J Neurosurg 1988; 69: 23946.

26) Kim P, Lorenz RR, Sundt TM Jr, et al. Release of endothelium-derived relaxing factor after subarachnoid hemorrhage. J Neurosurg 1989; 70: 108-14.

27) Kim P, Schini VB, Sundt TM Jr, et al. Reduced production of cGMP underlies the loss of endothelium-dependent relaxations in the canine basilar artery after subarachnoid hemorrhage. Circ Res 1992; 70: 248-55.

28) Xu A, Wang Y, Lam KSL, et al. Vascular actions of adipokines: molecular mechanisms and therapeutic implications. Adv Pharmacol 2010; 60: 229-56.

29) Hui X, Lam KSL, Vanhoutte PM, et al. Adiponectin and cardiovascular health: an update. Br J Pharmacol 2012; 165 : 574-90.

30) Vanhoutte PM. Effects of anoxia and glucose depletion on isolated veins of the dog. Am J Physiol 1976; 230: 1261-8. 
31) Van Nueten JM, Van Beek J, Vanhoutte PM. Inhibitory effect of lidoflazine on contractions of isolated canine coronary arteries caused by norepinephrine, 5-hydroxytryptamine, high potassium, anoxia and ergonovine maleate. J Pharmacol Exp Ther 1980; 213: 179-87.

32) De Mey JG, Vanhoutte PM. Heterogeneous behavior of the canine arterial and venous wall: importance of the endothelium. Circ Res 1982; 51: 439-47.

33) De Mey JG, Vanhoutte PM. Anoxia and endothelium dependent reactivity of the canine femoral artery. J Physiol 1983; 335: 65-74.

34) Rubanyi GM, Vanhoutte PM. Hypoxia releases a vasoconstrictor substance from the canine vascular endothelium. J Physiol 1985; 364: 45-56.

35) Félétou M, Huang Y, Vanhoutte PM. Endothelium-mediated control of vascular tone: COX-1 and COX-2 products. Br J Pharmacol 2011; 164: 894-912.

36) Félétou M, Kohler R, Vanhoutte PM. Nitric oxide: orchestrator of endothelium-dependent responses. Ann Med 2012; 44: 694-716.

37) Baretella O, Vanhoutte PM. Endothelium-dependent contractions: prostacyclin and endothelin-1, partners in crime? Adv Pharmacol 2016; 77: 177-208.

38) Gräser T, Vanhoutte PM. Hypoxic contraction of canine coronary arteries: role of endothelium and cGMP. Am J Physiol 1991; 261: H1769-77.

39) Pearson PJ, Lin PJ, Schaff HV, et al. Augmented endothelium-dependent constriction to hypoxia early and late fol- lowing reperfusion of the canine coronary artery. Clin Exp Pharmacol Physiol 1996; 23: 634-41.

40) Chan CKY, Mak J, Gao Y, et al. Endothelium-derived NO, but not cyclic GMP, is required for hypoxic augmentation in isolated porcine coronary arteries. Am J Physiol Heart Circ Physiol 2011; 301: H2313-21.

41) Beste KY, Seifert R. cCMP, cUMP, cTMP, cIMP and cXMP as possible second messengers: development of a hypothesis based on studies with soluble guanylyl cyclase $\alpha(1) \beta(1)$. Biol Chem 2013; 394: 261-70.

42) Chen Z, Zhang X, Ying L, et al. cIMP-synthesized by sGC as a mediator of hypoxic contraction of coronary arteries. Am J Physiol Heart Circ Physiol 2014; 307: H328-36.

43) Detremmerie CM, Chen Z, Li Z, et al. Endotheliumdependent contractions of isolated arteries to thymoquinone require biased activity of soluble guanylyl cyclase with subsequent cyclic IMP production. J Pharmacol Exp Ther 2016; 358: 558-68.

44) Gao Y, Vanhoutte PM. Tissues cIMPly do not lie. Naunyn Schmiedebergs Arch Pharmacol 2014; 387: 901-3.

45) Gao Y, Chen Z, Leung SWS, et al. Hypoxic vasospasm mediated by cIMP: when soluble guanylyl cyclase turns bad. J Cardiovasc Pharmacol 2015; 65: 545-8.

46) Leung SWS, Gao Y, Vanhoutte PM. 3',5'-cIMP as potential second messenger in the vascular wall. In: Seifert $\mathrm{R}$ ed. Non-canonical Cyclic Nucleotides. New York: Springer International Publishing AG, 2017: 209-28. 